doi: $10.1038 /$ nature 04844

\title{
Increased cell-to-cell variation in gene expression in ageing mouse heart
}

Rumana Bahar ${ }^{1}$, Claudia H. Hartmann ${ }^{2}$, Karl A. Rodriguez ${ }^{3}$, Ashley D. Denny ${ }^{3}$, Rita A. Busuttil ${ }^{1}$, Martijn E. T. Dollé ${ }^{4}$, R. Brent Calder ${ }^{1}$, Gary B. Chisholm ${ }^{3}$, Brad H. Pollock ${ }^{3}$, Christoph A. Klein ${ }^{2}$ and Jan Vijg ${ }^{1}$

1. Buck Institute for Age Research, Novato, California 94945, USA

2. Institut für Immunologie, Ludwig-Maximilians Universität, 80336 München, Germany

3. University of Texas Health Science Center, San Antonio, Texas 78245, USA

4. National Institute of Public Health and the Environment, 3720 BA

Bilthoven, The Netherlands

Correspondence to: Jan $\mathrm{Vijg}^{1}$ Correspondence and requests for materials should be addressed to J.V. (Email: jvijg@buckinstitute.org).

\begin{abstract}
The accumulation of somatic DNA damage has been implicated as a cause of ageing in metazoa ${ }^{1,2}$. One possible mechanism by which increased DNA damage could lead to cellular degeneration and death is by stochastic deregulation of gene expression. Here we directly test for increased transcriptional noise in aged tissue by dissociating single cardiomyocytes from fresh heart samples of both young and old mice, followed by global mRNA amplification and quantification of mRNA levels in a panel of housekeeping and heart-specific genes. Although gene expression levels already varied among cardiomyocytes from young heart, this heterogeneity was significantly elevated at old age. We had demonstrated previously an increased load of genome rearrangements and other mutations in the heart of aged mice ${ }^{3,4}$. To confirm that increased stochasticity of gene expression could be a result of increased genome damage, we treated mouse embryonic fibroblasts in culture with hydrogen peroxide. Such treatment resulted in a significant increase in cell-to-cell variation in gene expression, which was found to parallel the induction and persistence of genome rearrangement mutations at a lacZ reporter locus. These results underscore the stochastic nature of the ageing process, and could provide a mechanism for age-related cellular degeneration and death in tissues of multicellular organisms.
\end{abstract}

With the emergence of in situ techniques it has become possible to visualize mRNA levels in single cells. In situ methods have even allowed the visualization of specific sites of transcription of multiple genes simultaneously ${ }^{5}$. However, accurate quantification and the demonstration of significant differences in the expression levels of different mRNAs in cells freshly derived from mammalian tissues have, thus far, not been widely attempted ${ }^{6}$. Here we use a modification of a highly accurate, unbiased method ${ }^{7}$ to amplify global mRNA from enzymatically dissociated cardiomyocytes from young and old mouse heart and quantify transcript levels of arbitrarily chosen genes by real-time polymerase chain reaction (PCR). The genes selected were seven housekeeping genes, three heartspecific genes, two protease-encoding genes, and three mitochondrial genes 
Nature 441, 1011-1014(22 June 2006)

(Table 1). Because any gene had the potential to undergo random changes in its transcript level, we used different nuclear and mitochondrial genes as reference genes, reasoning that although this would not allow us to draw conclusions about absolute differences, our basic hypothesis of a significant age-related increase in cell-to-cell variation in gene expression remained perfectly testable. 
Nature 441, 1011-1014(22 June 2006)

Table 1 | Significance of age-related increase in gene expresssion variation

\begin{tabular}{|c|c|c|}
\hline Genes & Significance & Normalizers \\
\hline \multicolumn{3}{|c|}{ Housekeeping } \\
\hline \multirow[t]{2}{*}{ Gapdh } & $P<0.0001$ & Actb \\
\hline & $P<0.0001$ & $B 2 m$ \\
\hline \multirow[t]{2}{*}{ Actb } & $P<0.0001$ & Gapdh \\
\hline & $P<0.0001$ & $\mathrm{~B} 2 \mathrm{~m}$ \\
\hline \multirow[t]{2}{*}{$B 2 m$} & $P<0.0001$ & Gapdh \\
\hline & $P<0.0001$ & Actb \\
\hline \multirow[t]{3}{*}{ Tuba6 } & $P<0.0001$ & Gapdh \\
\hline & $P<0.0001$ & Actb \\
\hline & $P<0.0001$ & $B 2 m$ \\
\hline \multirow[t]{3}{*}{ Lmna } & $P=0.010$ & Gapdh \\
\hline & $P=0.1131$ & Actb \\
\hline & $P=0.1027$ & $B 2 m$ \\
\hline \multirow[t]{2}{*}{ Cox6a2 } & $P<0.0001$ & Cox7a1 \\
\hline & $P<0.0001$ & Myl2 \\
\hline \multirow[t]{2}{*}{ Cox7a1 } & $P<0.0001$ & Cox6a2 \\
\hline & $P<0.0001$ & Myl2 \\
\hline \multicolumn{3}{|c|}{ Heart-specific } \\
\hline \multirow[t]{3}{*}{ Actc1 } & $P<0.0001$ & Gapdh \\
\hline & $P<0.0001$ & Actb \\
\hline & $P<0.0001$ & $B 2 m$ \\
\hline \multirow[t]{3}{*}{ Lpl } & $P<0.0001$ & Gapdh \\
\hline & $P<0.0001$ & $A c t b$ \\
\hline & $P<0.0001$ & $B 2 m$ \\
\hline \multirow[t]{2}{*}{$M y / 2$} & $P<0.0001$ & Cox6a2 \\
\hline & $P<0.0001$ & Cox7a1 \\
\hline \multicolumn{3}{|l|}{ Proteases } \\
\hline \multirow[t]{3}{*}{ Cst3 } & $P<0.0001$ & Gapdh \\
\hline & $P<0.0001$ & Actb \\
\hline & $P<0.0001$ & $B 2 m$ \\
\hline \multirow[t]{3}{*}{ Ctss } & $P=0.017$ & Gapdh \\
\hline & $P=0.7761$ & Actb \\
\hline & $P=0.8073$ & $B 2 m$ \\
\hline \multicolumn{3}{|c|}{ Mitochondrial } \\
\hline \multirow[t]{2}{*}{ COX1 } & NS & $\cos 2$ \\
\hline & NS & $\operatorname{cox} 3$ \\
\hline \multirow[t]{2}{*}{$\operatorname{cox} 2$} & NS & $\operatorname{cox} 1$ \\
\hline & NS & $\operatorname{cox} 3$ \\
\hline \multirow[t]{2}{*}{$\operatorname{cox} 3$} & NS & $\operatorname{cox} 1$ \\
\hline & NS & $\operatorname{cox} 2$ \\
\hline
\end{tabular}

The significance of increased cell-to-cell variation in gene expresssion in young versus old mouse heart was determined using Bartlett's test. NS, not significant.

Heart cardiomyocytes were isolated from a similar area of ventricular heart tissue of young ( 6 months) and old (27 months) male mice. Dissociated cells contained over $90 \%$ rod-shaped, striated myocytes, which were easily recognizable under the microscope as cardiomyocytes (Fig. 1a). There was no evidence of the typical 
signs of either apoptotic or necrotic cell death-that is, rounded-up cells with plasma membrane blebs (apoptosis), or cell membrane disruption, extensive vacuolization or swollen nuclei (necrosis) ${ }^{8}$. Viability of virtually all cells from both young and old mice was also indicated by Trypan-blue dye exclusion.

\section{Figure 1: Reproducibility of unbiased, global mRNA amplification of mouse cardiomyocytes.}
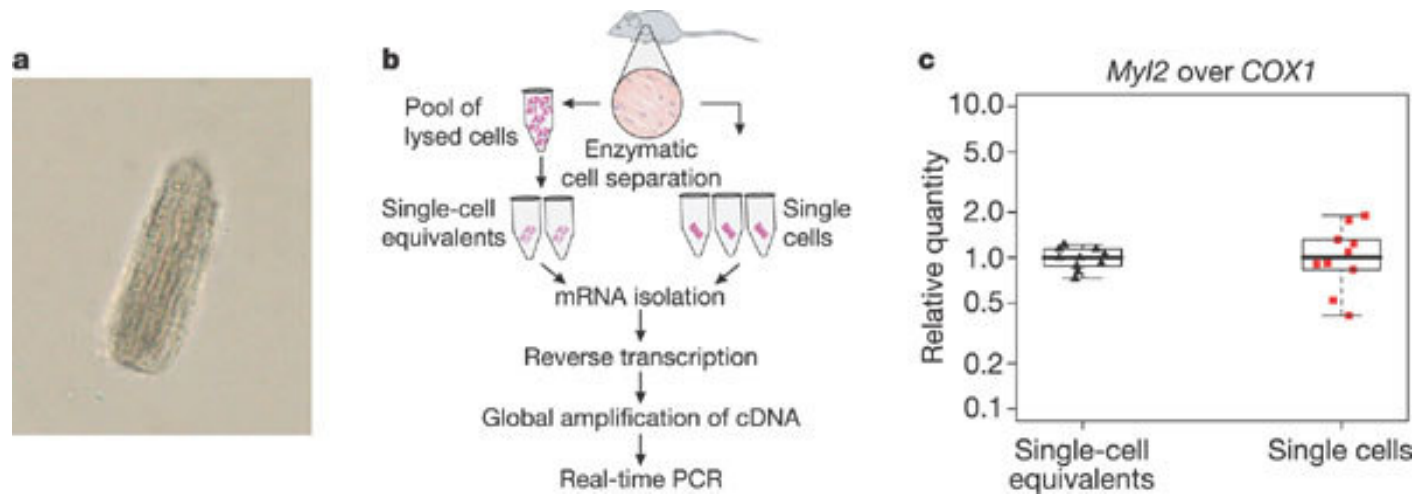

a, Typical morphology of cardiomyocytes after enzymatic dissociation. Original magnification, $\times 400$. b, Schematic representation of the strategy to validate cellto-cell variation in gene expression as compared with the experimental variation in repeated global mRNA amplification. The single-cell equivalents were made from a pool of lysed cardiomyocytes. Fifteen single cells and ten single-cell equivalents were separately subjected to mRNA isolation, reverse transcription and global cDNA amplification. c, Relative expression of the Myl2 gene (over COX1). Significantly increased variability was observed among the single cells as compared with the single-cell equivalents $(P=0.0026)$. Boxes in the box plots indicate the interquartile range (IQR) with the median; the whiskers indicate 1.5 xthe IQR.

To ensure that the expression of our target genes was stable during the isolation procedure, we compared heart tissue that was flash-frozen immediately after resection with a similar heart sample that was subjected to the isolation procedure-that is, sliced with a razor blade, incubated for 30 min with collagenase at room temperature $\left({ }^{\circ} 20^{\circ} \mathrm{C}\right)$ and then flash-frozen. A small amount of each tissue was amplified according to the same protocol. There was no significant effect of either the enzymatic treatment or the age of the animal on the expression levels of the target genes (Supplementary Table 1).

To verify the reproducibility of the amplification method in maintaining correct representation of individual mRNA levels, we repeatedly amplified the equivalent of a single cell from a pool of lysed cardiomyocytes, as compared with amplifications of single cells, from the heart of a 6-month-old mouse (Fig. 1b). We subsequently quantified for each cell, or single-cell equivalent, the expression level of the heart-specific gene Myl2, encoding the myosin ventricular regulatory light chain. In this case, the highly expressed mitochondrial cytochrome c oxidase gene COX1 (also known as $\mathrm{mt}-\mathrm{Co} 1$ ) was used as the reference gene. The results show very little variation in relative quantity among single-cell equivalents (Fig. 1c), which was within the range of the real-time PCR error. In contrast, variation in the relative quantity of Myl2 among single cells was significant, indicating cellto-cell variation in gene expression already occurs among cells from a young heart. Similar results were obtained with other highly expressed genes. Notably, transcriptional noise among single-cell equivalents was significant when studying 
genes expressed at a lower level in the heart, such as Actb or Gapdh, probably because of increased sensitivity of transcripts to stochastic fluctuations when present at low copy number ${ }^{9}$.

We then captured at least 15 individual cardiomyocytes for each of three young and three old animals, and subjected each cell to the global mRNA amplification procedure followed by quantification of the expression level of each target gene by real-time PCR. For normalization, we used different genes in the panel depending on the expression level (Table 1). Bartlett's test for unequal variances was used to test for differences in gene expression variability. The results indicated a highly significant increase in cell-to-cell variation in the expression levels of all nuclear genes in the old animals as compared with the young ones (Table 1). This increased cell-to-cell variation was observed irrespective of the reference gene used; however, for the genes Lmna and Ctss significance levels were reached with only one reference gene.

An example of the increased cell-to-cell variation in transcript levels in old mouse heart is shown in Fig. 2a for $\beta$-actin (Actb), $B-2$ microglobulin (B2m), lipoprotein lipase (Lpl) and cardiac $x$-actin (Actc1), normalized over glyceraldehyde-3phosphate dehydrogenase (Gapdh). In contrast, no significant cell-to-cell variation was observed when quantifying the three mitochondrial cytochrome $c$ oxidase genes-COX1, COX2 (also known as mt-Co2) and COX3 (also known as $\mathrm{mt}-\mathrm{Co} 3$ )-with either of these as the reference gene (Fig. 2b; Table 1). This is not unexpected, as these genes are transcribed as one long RNA molecule with many thousands of mitochondria present in each cardiomyocyte. When normalized against a nuclear gene, such as Gapdh, significant cell-to-cell variation in the relative quantities of each mitochondrial gene was always found (data not shown). These results underscore the stochasticity residing in nuclear genes and independently confirm the unbiased nature and reproducibility of the global mRNA amplification method. 
Figure 2: Increased cell-to-cell variation in gene expression among cardiomyocytes from the heart of old as compared with young mice.

a
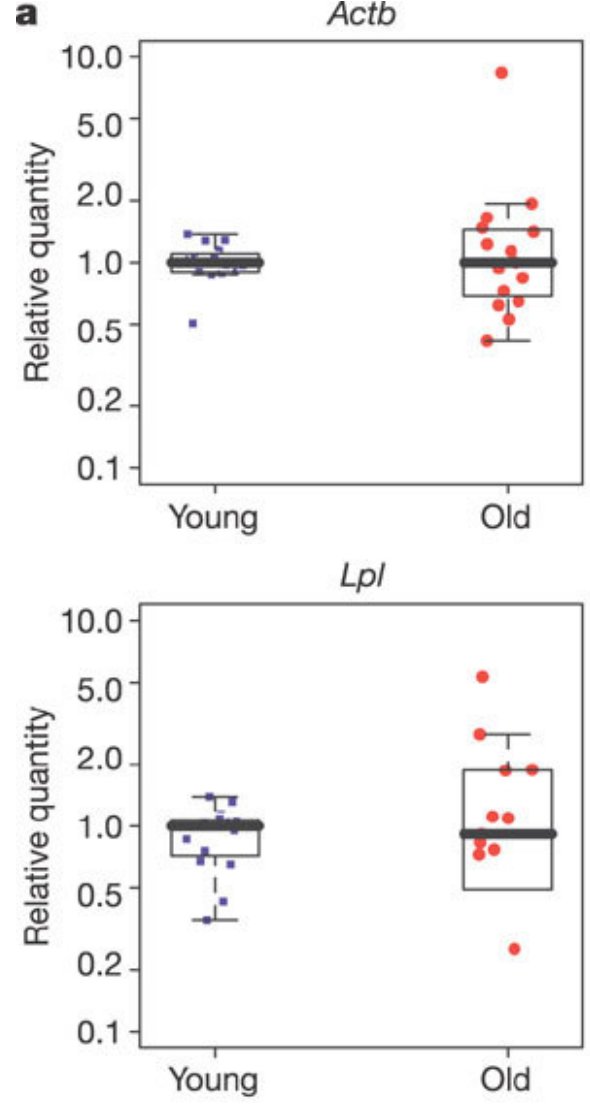

b

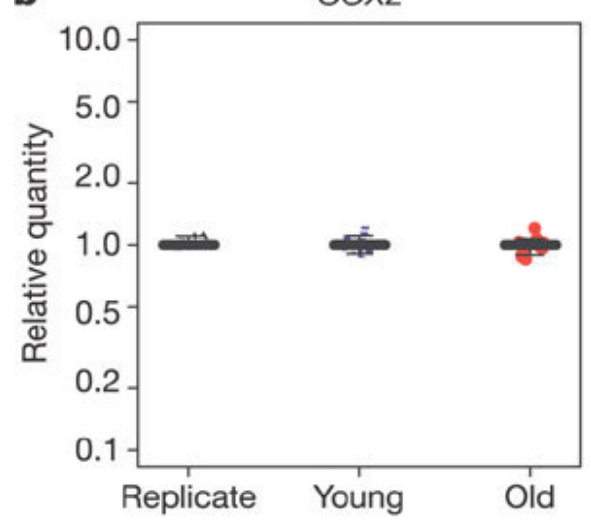

c

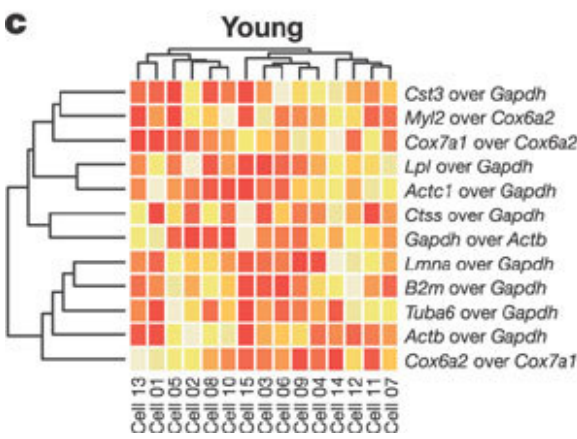

$B 2 m$

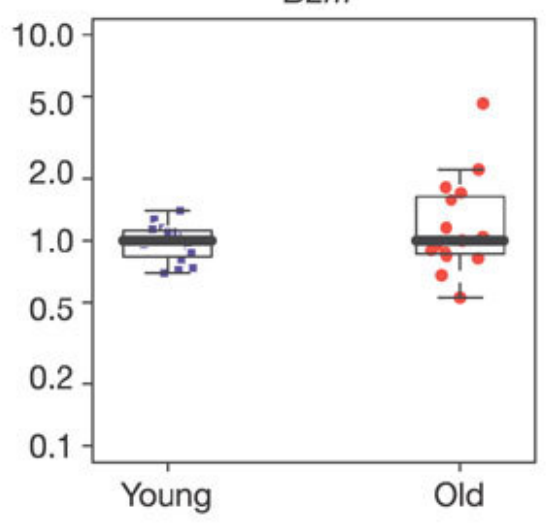

Actc1

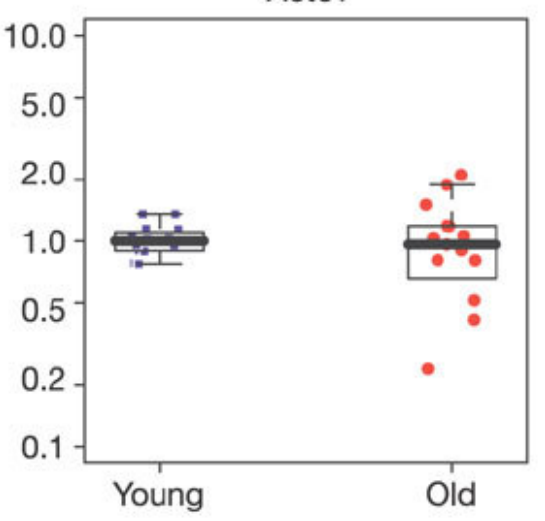

$\operatorname{cOX} 3$

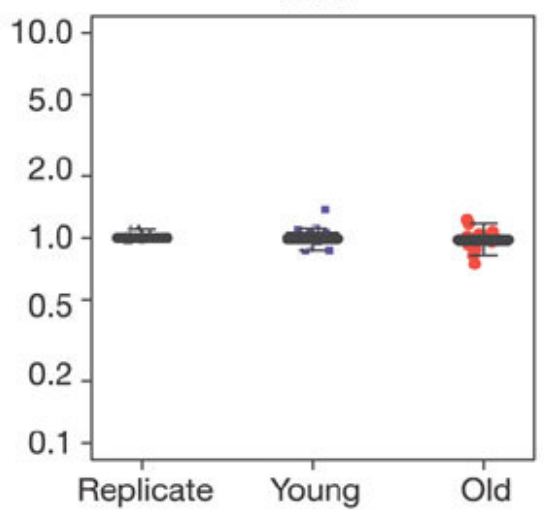


a, Examples of four genes showing statistically significant cell-to-cell variability in expression (Actb, B2m, Lpl and Actc1; normalized over Gapdh). P-values can be found in Table 1. Box plots are as in Fig. 1. b, Expression of mitochondrial cytochrome c oxidase genes (normalized over COX1) in the same cells showed no statistically significant increase in stochastic variation. Ten replicates from the same template were analysed along with the young and old cells to determine the real-time PCR error. Box plots are as in Fig. 1. c, Representative heat maps of one young and one old animal revealed no significant pattern of change in $\Delta \Delta \mathrm{C}_{T}$ (ranked for each gene) among the cells (red = low $\triangle A C_{T}$ levels; white = high $\triangle A C_{T}$ levels), indicating that the observed cell-to-cell variation is random.

We consider it unlikely that the observed cell-to-cell variation in relative gene expression and its increase with age are caused by the isolation procedure, because a doubling of collagenase concentration or incubation time resulted in the same range of cell-to-cell variation and a similar increase with age (data not shown). Nevertheless, we tested for a possible systematic effect on gene expression in the different cells by constructing heat plots for each group of cells-that is, from the three young and three old animals. The results show no specific correlation of the relative gene expression levels for any of the cells, allowing us to conclude that the observed variation is random and free of systematic bias (Fig. 2c).

Noise is inherent in the basic process of transcription, especially for genes expressed at low levels ${ }^{9,10}$. We considered the possibility that alterations in the somatic genome-for example, as induced by reactive oxygen species (a likely causal factor in ageing)-could contribute to increased stochasticity of gene expression. To confirm that genotoxic treatment does lead to increased stochasticity of gene expression in cells, we treated cultured mouse embryonic fibroblasts (MEFs) at early passage with $0.1 \mathrm{mM}$ hydrogen peroxide $\left(\mathrm{H}_{2} \mathrm{O}_{2}\right)$, a known generator of oxidative damage. At this dose the survival rate is approximately $80 \%$. At different time points after treatment, individual cells were obtained and global mRNA amplified, similar to the cardiomyocytes from young and old heart. For these cells, we assessed the mRNA levels of three housekeeping genes-that is, Actb, B2m and Tuba6 with Gapdh as the normalizer. As compared with single-cell equivalents, untreated cells showed a trend $(P=0.0847)$ towards cell-to-cell variation in gene expression, similar to the situation in cardiomyocytes from young animals, albeit with less magnitude (Fig. $3 a$ ). At $48 \mathrm{~h}$ after the treatment with $\mathrm{H}_{2} \mathrm{O}_{2}$, cell-to-cell variation in gene expression greatly increased. This is shown in Fig. 3a for Actb as an example, but a similar increase was observed for B2m and Tuba6 (data not shown). 
Figure 3: $\mathrm{H}_{2} \mathrm{O}_{2}$ treatment of MEFs increases cell-to-cell variation in gene expression in parallel to the induction of genome rearrangements.
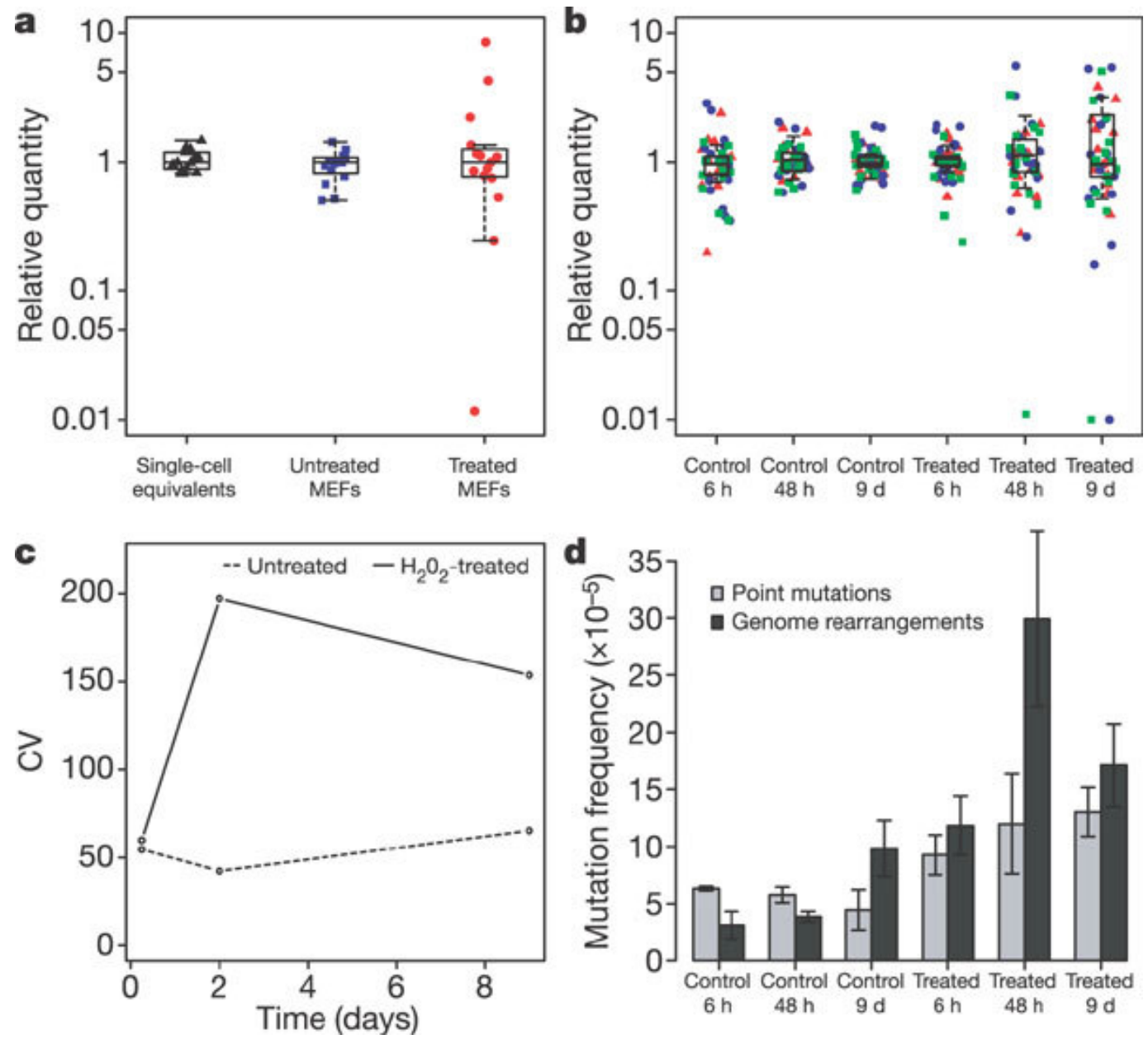

a, Cell-to-cell variation in relative expression of Actb (normalized over Gapdh) among MEF single-cell equivalents, untreated MEFs, and MEFs at $48 \mathrm{~h}$ after treatment with $0.1 \mathrm{mM} \mathrm{H}_{2} \mathrm{O}_{2}$. The variation among the treated cells is significantly greater than among the untreated cells $(P<0.0001)$. Box plots are as in Fig. 1. b, Cell-to-cell variation in relative expression of Actb (red), B2m (blue) and Tuba6 (green) (normalized over Gapdh) at $6 \mathrm{~h}, 48 \mathrm{~h}$ and 9 days after treatment with $\mathrm{H}_{2} \mathrm{O}_{2}$. Both at $48 \mathrm{~h}$ and at 9 days, cell-to-cell variation in relative expression of the three genes was significantly greater than in the controls $(P<0.0001$ in both cases). At $6 \mathrm{~h}$, variation among the untreated, control cells was somewhat higher than among the treated cells $(P=0.0382)$, but only in this experiment. Box plots are as in Fig. 1. C, Coefficient of variation (CV) calculated for all relative quantities of Actb, B2m and Tuba6 at each time point and treatment condition. The $6 \mathrm{~h}, 48 \mathrm{~h}$ and 9 day time points are based on 2, 4 and 3 independent experiments, respectively. Each experiment consists of 11-15 single-cell determinations for each gene under each treatment condition. d, lacZ mutation frequencies at $6 \mathrm{~h}, 48 \mathrm{~h}$ and 9 days after $\mathrm{H}_{2} \mathrm{O}_{2}$ treatment as compared with control populations. Mutation frequencies are averages from three independent determinations from each of two parallel experiments. The subdivision into point mutations (grey bars) and genome rearrangements (black bars) was made on the basis of restriction enzyme analysis of 48 mutants taken from each experiment. Error bars indicate s.d. 
To investigate when this $\mathrm{H}_{2} \mathrm{O}_{2}$-induced transcriptional noise occurred and how long it would last, we studied cell-to-cell variation in the expression of these three housekeeping genes at $6 \mathrm{~h}, 48 \mathrm{~h}$ and 9 days after treatment in several independent experiments. The results of one such experiment is shown in Fig. 3b. At $6 \mathrm{~h}$ after treatment, cell-to-cell variation in the relative expression level of none of these genes was increased. In fact, untreated cells at this early stage sometimes showed a somewhat higher variation, for which we have no immediate explanation. This result strongly suggests that the increased transcriptional noise induced by $\mathrm{H}_{2} \mathrm{O}_{2}$ is not due to direct chemical damage of DNA, proteins or lipidsthe effects of which are likely to appear almost immediately.

At $48 \mathrm{~h}$ after treatment, the increased stochasticity observed in the earlier experiment was reproduced. As documented by numerous investigators, $\mathrm{H}_{2} \mathrm{O}_{2}$ induces replicative senescence in primary mouse or human fibroblasts ${ }^{11}$. At $48 \mathrm{~h}$ after treatment, the cell populations started to show an increased number of senescent cells, characterized by their flattened and enlarged morphology (data not shown). During this period, when the cells were maintained in the same culture plates, cell death was not apparent, and at 9 days after treatment almost all cells were senescent. At that time, the increased cell-to-cell variation in gene expression among the $\mathrm{H}_{2} \mathrm{O}_{2}$-treated populations was still highly significant (Fig. 3b). In Fig. 3c, we plotted the coefficients of variation at the three time points as averages of 2-4 independent experiments for the three genes together. The kinetics of the process indicates an increase at $48 \mathrm{~h}$, after which time the transcriptional noise seemed to remain stable. On the basis of these results, we conclude that $\mathrm{H}_{2} \mathrm{O}_{2}$-induced transcriptional noise is a relatively late event, which is virtually absent during the first hours after treatment. However, at least in a population of low cell-turnover, the induced transcriptional noise is persistent, although a modest decline at 9 days cannot be ruled out.

As the $\mathrm{H}_{2} \mathrm{O}_{2}$-induced transcriptional noise is unlikely to be caused by an early event, such as DNA damage or damage to proteins or lipids, we analysed mutations at a lac $Z$ reporter gene as a relatively late marker for genomic instability. The lacZ reporter genes were present in the C57BL/ 6 mouse line from which the cells had been derived ${ }^{12}$. The results of this analysis indicate that although some mutations were already present at $6 \mathrm{~h}$ after treatment, their frequency increased substantially at $48 \mathrm{~h}$ and declined somewhat at 9 days. Most of these mutations were large genome rearrangements, as indicated by restriction analysis of the mutant lac $Z$ plasmids recovered from the cells (Fig. $3 d$ ). Hence, the kinetics of $\mathrm{H}_{2} \mathrm{O}_{2}$-induced mutations and transcriptional noise are quite similar, though not exactly the same.

Although it is tempting to suggest that the accumulation of genome rearrangements, which we previously demonstrated to accumulate with age in the mouse heart ${ }^{3,4}$, is a cause of increased stochasticity of gene expression in ageing-for example, through gene dose and/or position effects-random changes in DNA methylation or chromatin remodelling could contribute as well. However, other cellular changes unrelated to genome alterations cannot be excluded at this stage. Stochastic changes in gene expression have been considered as a possible cause of ageing since Orgel proposed his 'error catastrophe' theory ${ }^{13}$, and the role of chance events in ageing is now increasingly appreciated ${ }^{14,15,16}$. Our present results do not allow any further conclusions with respect to increased transcriptional noise as a mechanism of ageing. However, stochasticity is ubiquitous in biological systems and increased noise in gene expression has been implicated in reduced organismal fitness ${ }^{17}$. It is conceivable that a variety of persistent forms of damage to biological macromolecules could initiate a gradual increase in transcriptional noise introducing phenotypic variation among cells. 
Such variation could generally become detrimental to normal cell functioning, which would explain many aetiological characteristics of the ageing process, most notably the highly variable, progressive decline in organ function.

\section{Methods}

See Supplementary Methods for detailed experimental procedures.

\section{Animals and tissue collection}

Young (6 months) and old (27 months) BALB/c mice were obtained from the National Institute on Aging and used after two weeks. Animals were euthanized by $\mathrm{CO}_{2}$ and the hearts perfused with PBS and excised. The ventricular parts were subsequently dissected and either flash frozen and stored at $-80^{\circ} \mathrm{C}$ or immediately used for single-cell isolation.

\section{Isolation of cardiomyocytes}

The ventricular tissue was minced and incubated for $30 \mathrm{~min}$ at room temperature $\left(\approx 20^{\circ} \mathrm{C}\right)$ under gentle shaking in collagenase in Krebs-Henseleit buffer. Single cells were collected under an inverted microscope by hand-held capillaries, deposited in PCR tubes and immediately frozen on dry ice and stored at $-80{ }^{\circ} \mathrm{C}$ until needed. The viability of the cardiomyocytes was determined by Trypan-blue dye exclusion and found to be over $90 \%$ for both young and old mice.

\section{Isolation of mouse embryonic fibroblasts and treatment with $\mathrm{H}_{2} \mathrm{O}_{2}$}

Mouse embryonic fibroblasts (MEFs) were isolated from embryonic day 13.5 embryos of C57BL/6 mice harbouring multiple copies of chromosomally integrated plasmids containing the lacZ mutational reporter gene, as described ${ }^{18}$. For each experiment, a total of 1 million cells were seeded in a $10-\mathrm{cm}$ culture dish and treated with $0.1 \mathrm{mM} \mathrm{H}_{2} \mathrm{O}_{2}$ (Sigma) in medium without serum for $2 \mathrm{~h}$ at $37^{\circ} \mathrm{C}$.

\section{Global mRNA amplification}

mRNA isolation and global amplification of first-strand cDNA was carried out according to a modified version of the procedure described in ref. 7. For each young versus old animal pair, cells were processed simultaneously using the same reagents.

\section{Real-time PCR}

Gene expression levels were quantified by real-time PCR, using the ABI Prism $7900 \mathrm{HT}$ system (Applied Biosystems). A list of the panel of genes tested along with the primer sequences used is provided in Supplementary Table 1.

\section{Detection and analysis of genomic mutations}

DNA was extracted from treated and control cells, and mutant frequencies and spectra were determined as described ${ }^{19}$. For each sample, at least 48 mutants were characterized. 


\section{Statistical analysis}

For each target gene studied, the expression level relative to that of the reference gene and calibrator ( $\triangle A \mathrm{C}_{T}$; see Supplementary Methods for further definition) was modelled as a linear function of mouse age. Bartlett's tests were used to determine if the variance of young and old mice differed significantly.

\section{Acknowledgments}

This work was supported by an NIH grant to J.V. and a BioFuture grant from the German Federal Ministry for Education and Science to C.A.K. We thank K. Khrapko and R. Beems for advice and useful suggestions. Author Contributions J.V. conceived, designed and supervised the study (initially with M.E.T.D.), obtained the funding and took the primary role in writing the paper. R.B. designed and performed the experiments, assisted by K.A.R., A.D.D. and R.A.B. R.B.C., G.B.C. and B.H.P. performed statistical analyses and made the figures. C.H.H. and C.A.K. provided the modified protocol for global mRNA amplification.

\section{Competing interests statement:}

The authors declared no competing interests.

\section{Supplementary informationaccompanies this paper.}

\section{References}

1. Vijg, J. \& Dollé, M. E. Large genome rearrangements as a primary cause of aging. Mech. Ageing Dev. 123, 907-915 (2002)

2. Kirkwood, T. B. Understanding the odd science of aging. Cell 120, 437-447 (2005)

3. Dollé, M. E. , Snyder, W. K. , Gossen, J. A. , Lohman, P. H. \& Vijg, J. Distinct spectra of somatic mutations accumulated with age in mouse heart and small intestine. Proc. Natl Acad. Sci. USA 97, 8403-8408 (2000)

4. Dollé, M. E. \& Vijg, J. Genome dynamics in aging mice. Genome Res. 12, 1732-1738 (2002)

5. Levsky, J. M. , Shenoy, S. M. , Pezo, R. C. \& Singer, R. H. Single-cell gene expression profiling. Science 297, 836-840 (2002)

6. Levsky, J. M. \& Singer, R. H. Gene expression and the myth of the average cell. Trends Cell Biol. 13, 4-6 (2003)

7. Klein, C. A. et al. Combined transcriptome and genome analysis of single micrometastatic cells. Nature Biotechnol. 20, 387-392 (2002)

8. Kang, P. M. , Haunstetter, A. , Aoki, H. , Usheva, A. \& Izumo, S. Morphological and molecular characterization of adult cardiomyocyte apoptosis during hypoxia and reoxygenation. Circ. Res. 87, 118-125 (2000)

9. Kaern, M. , Elston, T. C. , Blake, W. J. \& Collins, J. J. Stochasticity in gene expression: from theories to phenotypes. Nature Rev. Genet. 6, 451-464 (2005)

10. Becskei, A. , Kaufmann, B. B. \& van Oudenaarden, A. Contributions of low molecule number and chromosomal positioning to stochastic gene expression. Nature Genet. 37, 937-944 (2005)

11. Parrinello, S. et al. Oxygen sensitivity severely limits the replicative lifespan of murine fibroblasts. Nature Cell Biol. 5, 741-747 (2003)

12. Boerrigter, M. E. , Dollé, M. E. , Martus, H. J. , Gossen, J. A. \& Vijg, J. Plasmid-based transgenic mouse model for studying in vivo mutations. Nature 377, 657-659 (1995)

13. Orgel, L. E. The maintenance of the accuracy of protein synthesis and its relevance to ageing. Proc. Natl Acad. Sci. USA 49, 517-521 (1963)

14. Finch, C. E. \& Kirkwood, T. B. Chance, Development, and Aging (Oxford Univ. Press, New York, 2000)

15. Rea, S. L. , Wu, D. , Cypser, J. R. , Vaupel, J. W. \& Johnson, T. E. A stress-sensitive reporter predicts longevity in isogenic populations of Caenorhabditis elegans. Nature Genet. 37, 894898 (2005)

16. Golden, T. R. \& Melov, S. Microarray analysis of gene expression with age in individual nematodes. Aging Cell 3, 111-124 (2004)

17. Fraser, H. B. , Hirsh, A. E. , Giaever, G. , Kumm, J. \& Eisen, M. B. Noise minimization in eukaryotic gene expression. PLoS Biol. 2, e137 (2004) 
Nature 441, 1011-1014(22 June 2006)

18. Busuttil, R. A. , Rubio, M. , Dollé, M. E. , Campisi, J. \& Vijg, J. Oxygen accelerates the accumulation of mutations during the senescence and immortalization of murine cells in culture. Aging Cell 2, 287-294 (2003)

19. Dollé, M. E. , Martus, H. J. , Gossen, J. A. , Boerrigter, M. E. \& Vijg, J. Evaluation of a plasmid-based transgenic mouse model for detecting in vivo mutations. Mutagenesis 11, 111-118 (1996) 